\title{
Cardiac magnetic resonance imaging for the assessment of the heart and pulmonary circulation in pulmonary hypertension
}

\author{
L.E.R. McLure and A.J. Peacock
}

ABSTRACT: Pulmonary hypertension is a disease of the pulmonary arteries resulting in a progressive increase in pulmonary vascular resistance, ultimately leading to right ventricular failure and death. The functional capacity of the right ventricle is a major prognostic determinant. Our understanding of right ventricle performance in pulmonary hypertension has been hindered by the lack of techniques that give a reliable picture of right ventricle morphology and function. Cardiac magnetic resonance (CMR) imaging enables a unique combination of morphological and functional assessment of the right ventricle and pulmonary circulation. In this review article, we introduce the technique of CMR imaging, review its use in imaging of the heart and pulmonary circulation and discuss its current and future application to the management of patients with pulmonary hypertension.

There have been recent major advances in our understanding of the mechanism of disease development, in the diagnostic process, and in the treatment of pulmonary hypertension. Therapeutic advances in the management have reinforced the requirement for noninvasive, accurate and reproducible methods of assessment to act as "end-points" to measure the effects of treatment. We anticipate CMR imaging will increasingly be utilised as the primary modality for combined anatomic and functional assessments that enable more complete and efficient evaluation of pulmonary hypertension patients.

KEYWORDS: Cardiac magnetic resonance imaging, pulmonary circulation, pulmonary hypertension, right heart

\footnotetext{
$\mathrm{n}$ the present article, it is our aim to introduce the technique of cardiovascular magnetic resonance imaging, to review its use in imaging of the heart and pulmonary circulation and to discuss its current and future application to the management of patients with pulmonary hypertension. This paper is part of a series of articles published in the European Respiratory Journal summarising and commenting on the latest developments in pulmonary vascular disease (see footnote).
}

\section{PULMONARY HYPERTENSION}

Pulmonary hypertension is a disease of the pulmonary arteries that is characterised by vascular proliferation and remodelling [1, 2]. It results in a progressive increase in pulmonary vascular resistance (PVR) and ultimately, right ventricular failure and death. The diagnostic classification of pulmonary hypertension is described in table 1 . This current classification was established during the 2003 World Symposium on Pulmonary Hypertension [3].

Previous articles in this series: No. 1: Dupuis J, Hoeper MM. Endothelin receptor antagonists in pulmonary arterial hypertension. Eur Respir J 2008; 31 : 407-415. No. 2: Gomberg-Maitland M, Olschewski H. Prostcyclin therapies for the treatment of pulmonary arterial hypertension. Eur Respir J 2008; 31: 891-901. No. 3: Behr J, Ryu JH. Pulmonary hypertension in interstitial lung disease. Eur Respir J 2008; 31: 1357-1367. No. 4: Wilkins MR, Wharton J, Grimminger F, Ghofrani HA. Phosphodiesterase inhibitors for the treatment of pulmonary hypertension. Eur Respir J 2008; 32: 198-209. No. 5: Warwick G. Thomas PS, Yates DH. Biomarkers in pulmonary hypertension. Eur Respir J 2008; 32: 503-512. No. 6: Chaouat A, Naeije R, Weitzenblum E. Pulmonary hypertension in COPD. Eur Respir J 2008; 32: 1371-1385. No. 7: Montani D, Price LC, Dorfmuller P, et al. Pulmonary veno-occlusive disease. Eur Respir J 2009; 33: 189-200. No. 8: Faughnan ME, Granton JT, Young LH. The pulmonary vascular complications of hereditary haemorrhagic telangiectasia. Eur Respir J 2009; 33: $1186-1194$.

European Respiratory Journal Print ISSN 0903-1936 Online ISSN 1399-3003

\section{AFFILIATIONS}

Scottish Pulmonary Vascular Unit, Golden Jubilee National Hospital, Glasgow, UK

\section{CORRESPONDENCE}

A.J. Peacock

Scottish Pulmonary Vascular Unit Golden Jubilee National Hospital Beardmore Street

Clydebank

G81 4HX

West Dumbartonshire

Scotland, UK

E-mail: SPVUnit@gjnh.scot.nhs.uk

Received:

October 232007

Accepted after revision:

December 232008

STATEMENT OF INTEREST

A statement of interest for A.J.

Peacock can be found at

www.erj.ersjournals.com/misc/ statements.dtl 
TABLE 1 Diagnostic classification of pulmonary hypertension (Venice 2003)

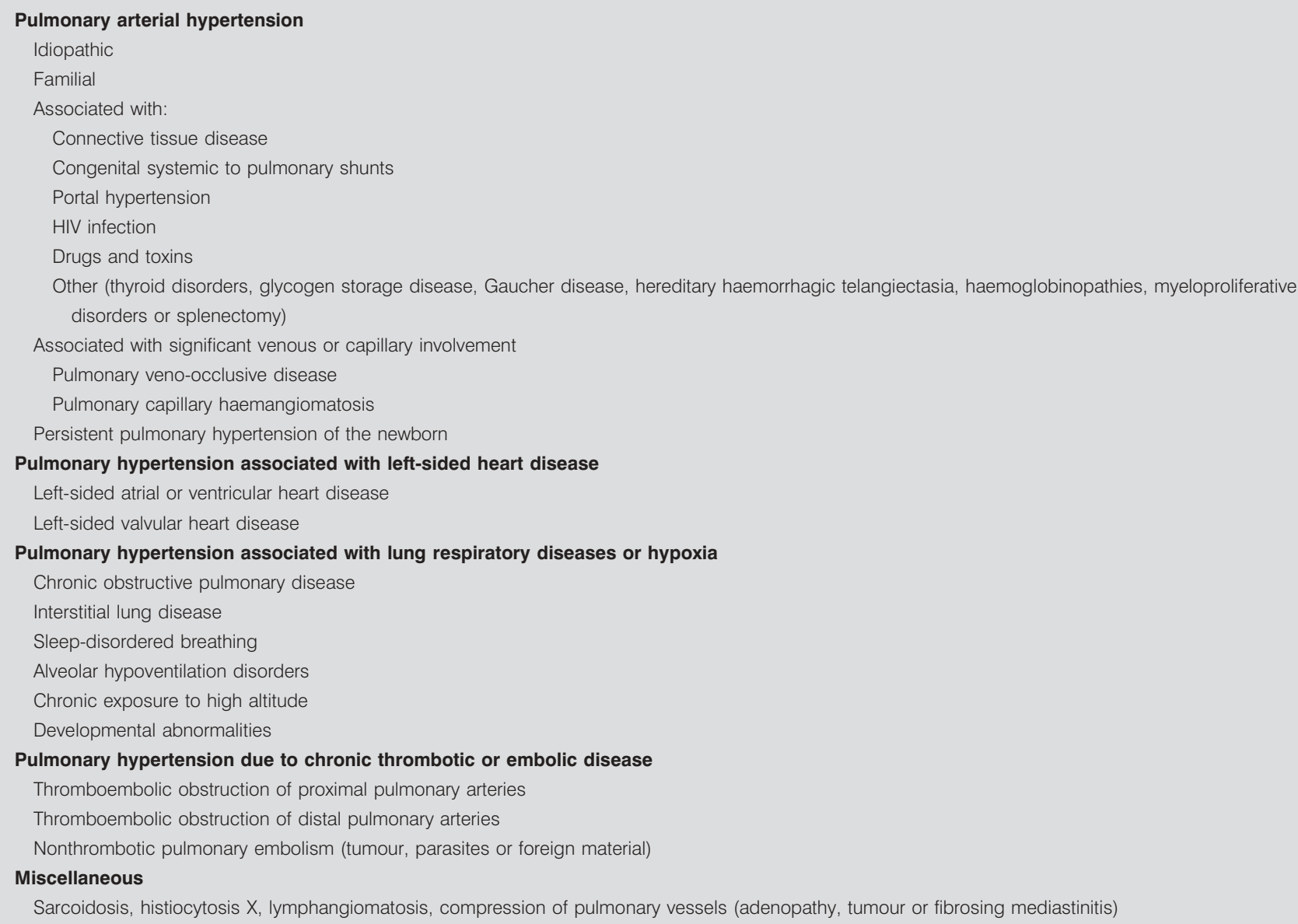

The functional capacity of the right ventricle is a major prognostic determinant in pulmonary hypertension. It is unknown why some patients with markedly elevated pulmonary artery pressure $(P \mathrm{pa})$ maintain well-preserved cardiac function for several years, while others with equal or less severe pulmonary hypertension suffer rapidly progressive right heart failure. One factor that has hindered the understanding of right ventricular performance in patients with pulmonary hypertension has been a lack of techniques that give a reliable picture of right ventricular morphological and functional change in the face of increasing outflow obstruction.

\section{ASSESSMENT OF THE RIGHT VENTRICLE AND PULMONARY CIRCULATION}

\section{Anatomy of the right ventricle}

The right ventricle is characterised by a crescent-like shape and a thin wall. The right ventricle pumps the same stroke volume as the left ventricle but with $\sim 25 \%$ of the stroke work because of the low resistance of the pulmonary vasculature. Normally, the right ventricle has one-sixth of the muscle mass and performs against one-tenth of the vascular resistance compared to the left ventricle. Of note, longitudinal shortening is a greater contributor to right ventricular stroke volume than short-axis (circumferential) shortening [4]. In contrast to the symmetrical shape of the left ventricle, right ventricle geometry is complex. The normal right ventricle has an inflow component formed by the atrioventricular septum, tricuspid valve and subvalvular apparatus, an apical trabecular component and an outflow tract that continues into the pulmonary trunk. The right ventricular inflow and outflow regions are separated by the crista ventricularis and the right ventricle is "wrapped around" the left ventricle. This shape and orientation makes the evaluation of right ventricular volumes, systolic function and myocardial mass difficult using two-dimensional cross-sectional imaging modalities, such as echocardiography.

\section{Assessment of the right ventricle}

Several imaging modalities are available for the assessment of the right ventricle.

1) Echocardiography is the most well established imaging technique for screening and diagnosis of pulmonary hypertension [5]. As an imaging modality, it has the advantage of being widely available, inexpensive and safe. Echocardiography provides a quantitative estimate of systolic $P$ pa, using the peak velocity of the regurgitant jet through the tricuspid valve. It provides an assessment of associated anatomical abnormalities, e.g. evidence of congenital heart disease and right ventricle enlargement. This investigation relies upon geometric assumptions that can be difficult to adopt for the right ventricle, which has a complex shape. Limiting factors include 
operator dependence and influence by prevailing conditions, such as heart rate and body habitus. Most studies report a high correlation (0.57-0.93) between echocardiography and right heart catheterisation (RHC) measurements of systolic Ppa [6], although ARCASOY et al. [7] concluded that estimation of systolic Ppa by echocardiography is frequently inaccurate in patients with advanced lung disease.

2) Radionuclide ventriculography can be used to image the right heart, although exposure to ionising radiation is a disadvantage. Attenuation artefacts are common, which makes it difficult to delineate the right atrium and right ventricle accurately.

3) Multi-slice computed tomography (CT) can assess all morphological features of right heart adaptation and failure in pulmonary hypertension. Modern contrast-enhanced CT studies permit delineation of the cardiac chambers, valves, great cardiac vessels and even the coronary artery lumen. Because data acquisition in spiral multi-detector $\mathrm{CT}$ is continuous, retrospective ECG gating allows for image reconstruction in any phase of the cardiac cycle. Thus, endsystolic and end-diastolic images can be produced to assess ventricular volumes and function. However, radiation exposure is a limitation and it is therefore doubtful that CT would be the modality of choice for assessment of the right heart, especially for follow-up examinations.

\section{Assessment of the pulmonary circulation}

The following imaging techniques are used for the assessment of the pulmonary circulation.

1) Ventilation/perfusion $\left(V^{\prime} / Q^{\prime}\right)$ lung scans may be entirely normal in some patients with PHT. Small peripheral nonsegmental defects in perfusion are often present, which are normally ventilated ( $V^{\prime} / Q^{\prime}$ mismatch). Lung $V^{\prime} / Q^{\prime}$ scanning provides a means of diagnosis of chronic thromboembolic pulmonary hypertension (CTEPH). Typically, the perfusion defects are found in lobar and segmental regions leading to segmental defects in the perfusion image, which are normally ventilated (unmatched $V^{\prime} / Q^{\prime}$ defects). $V^{\prime} / Q^{\prime}$ scanning showed a sensitivity of $90-100 \%$ with specificity of $94-100 \%$ for distinguishing between idiopathic pulmonary hypertension and CTEPH [8]. Exposure to ionising radiation is a disadvantage of this technique.

2) Contrast-enhanced spiral CT of the lungs is indicated in pulmonary hypertension patients when the $V^{\prime} / Q^{\prime}$ scan is suggestive of segmental or subsegmental perfusion defects with normal ventilation. CT features of CTEPH include complete occlusion of pulmonary arteries, eccentric filling defects consistent with thrombi, recanalisation and stenoses or webs. Patients are exposed to ionising radiation and the risks of intravenous contrast agents during this investigation. Further limitations include the inability to perform dynamic images and flow measurements.

3) Traditional pulmonary angiography is required to identify patients with CTEPH who may benefit from pulmonary endarterectomy [8]. Pulmonary angiography is more accurate in the identification of distal obstructions. However, contrast angiography is an invasive procedure, with exposure to ionising radiation and contrast agents.

\section{Right heart catheterisation}

A diagnosis of pulmonary hypertension must be confirmed by RHC, which remains the gold standard for assessment of pulmonary haemodynamics. RHC provides direct and accurate measurements of $P$ pa, cardiac output and, hence, PVR. There are disadvantages to this procedure, but most important are the significant risks to the patient. A recent study by HOEPER et al. [9] assessed the risks associated with RHC $(7,218$ procedures) in patients with pulmonary hypertension. It was concluded that when performed in experienced centres, RHC in this patient group was associated with low morbidity and mortality rates (76 serious adverse events). Four fatal events were recorded in association with any of the catheter procedures, resulting in an overall procedure-related mortality of $0.055 \%$ (95\% CI $0.01-0.099 \%$ ). Apart from the risks, hospital admission is usually a procedure that may be inconvenient for patients. At present, RHC is required to confirm the diagnosis of pulmonary hypertension, to assess the severity of the haemodynamic impairment and to test the vasoreactivity of the pulmonary circulation. Some units also use RHC to determine success or response to treatment.

\section{CARDIAC MAGNETIC RESONANCE IMAGING Introduction}

Cardiac magnetic resonance (CMR) imaging is well established in clinical practice for the diagnosis and management of a wide spectrum of cardiovascular disease. Its advancing role is related to technical improvements, which allow increasingly rapid and robust data acquisition. Use of CMR represents the specialised application of magnetic resonance to the cardiovascular system, employing specialised receiver coils, pulse sequences and gating methods. Images may be performed with ECG gating/triggering and with respiratory suppression (breath-holding or navigator gating), thereby reducing image artefacts.

CMR is fundamentally safe. No short- or long-term ill effects have been reported at current field strengths $(<3 \mathrm{~T})$. Magnetic resonance does not interfere with the electron shells involved in chemical binding (e.g. DNA) that can be altered by ionising radiation. The phenomenon of magnetic resonance is restricted to atomic nuclei with unpaired spin, e.g. hydrogen, carbon, oxygen, sodium, potassium and fluorine. The majority of clinical CMR imaging involves the hydrogen nucleus, which is abundant in water, fat and muscle.

\section{Limitations of CMR}

CMR is expensive, not widely available and requires significant operator expertise. It can be a difficult examination for pulmonary hypertension patients to complete owing to time duration and breath-holding requirements. Claustrophobia is a significant problem. This can be overcome in the majority of patients by using mild sedation, although this is often inappropriate in pulmonary hypertension patients. Ferromagnetic objects must not enter the magnetic resonance scanner area, because they will become projectiles. This is an extremely important safety issue. Common practice is to specifically check and verify that each medical device present in patients is magnetic resonance compatible. The radiofrequency field, which is used for excitation, can induce heating of tissue and implanted devices. It is possible to 
stimulate sensitive tissues such as peripheral nerves owing to the rapidly changing gradient magnetic fields used to generate images. Myocardial stimulation has not been described with current hardware.

\section{Ventricular morphology and function by CMR}

Magnetic resonance imaging (MRI) produces tomographic still images that can accurately and reproducibly assess left ventricular and right ventricular chamber sizes, wall thickness and mass. The multifaceted nature of MRI enables it to be used not only for morphological assessment, but also for functional assessment. Conventional gradient-recalled echo or steadystate free-precession pulse (SSFP) sequences can be used to construct a cine image, which is a movie of 15-20 frames in which the full cardiac cycle can be seen; each movie frame represents $30-40 \mathrm{~ms}$ of the cycle. Recent technological advances enable the implementation of SSFP sequences which provide a substantially higher signal-to-noise ratio than can be obtained by conventional gradient-echo techniques. The contrast between myocardium and cavity blood [10] make planimetry of the interface accurate and easily reproducible for assessment of left and right ventricular function. The SSFP technique is the preferred CMR pulse sequence for acquisition of volumetric datasets of the left and right ventricles. Cine mode MRI allows regional and global systolic function to be evaluated because wall motion abnormalities can be identified. Ventricular volumes, ejection fraction and myocardial mass are usually obtained from a stack of contiguous "bright blood" cine CMR 5-10 mm slices covering the left and right ventricles acquired in short-axis or transverse orientation. Endocardial and epicardial contours are drawn during post-processing on end-diastolic and end-systolic frames, and left and right ventricular volumes are calculated as the sum of individual slice volumes (fig. 1). Ventricular mass is the product of myocardial volume and muscle-specific density $\left(1.05 \mathrm{~g} \cdot \mathrm{cm}^{-3}\right)$. A previous criticism of this technique has been the time required to analyse the cine data to generate accurate volume and mass data. New PC-based software solutions with intensity-based thresholding for semiautomated myocardial blood border definition has enabled analysis to become less time-consuming.

Impressive results for accuracy have been demonstrated by several investigators in various disease states [11-15]. The interstudy reproducibility of CMR-derived parameters of ventricular function and mass is good for both the left and right ventricles and is superior to two-dimensional and Mmode echocardiography [16-18]. The results from a study performed by GROTHUES et al. [19] demonstrate that the interstudy reproducibility of the right ventricle is lower than for the left ventricle, although CMR is still a reliable method and can be considered the gold standard for serial assessment of right ventricular volumes, function and mass.

\section{Flow analysis}

Phase contrast velocity mapping is an magnetic resonance sequence used to measure velocity and flow in blood vessels, or within the heart, in which each pixel in the image displays the signal phase, which is encoded. Volumetric flow (in millilitres per second) is obtained in each time frame by multiplying the spatial mean velocity (in centimetres per second) of blood flow with the cross-sectional area of the vessel

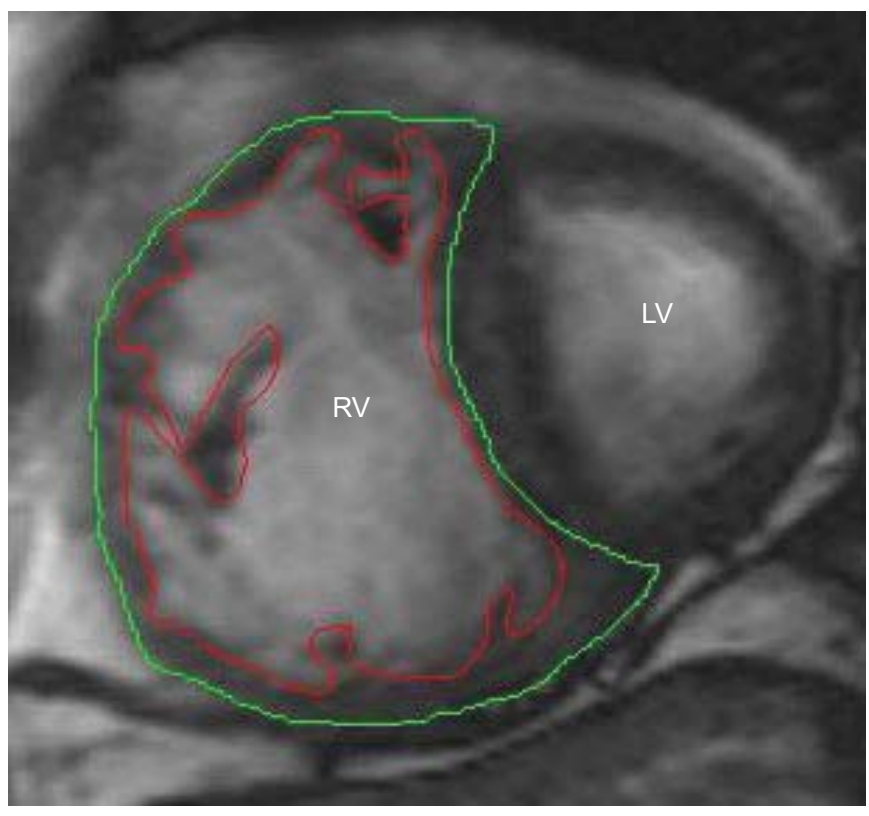

FIGURE 1. Planimetry of right ventricle (RV). Epicardial and endocardia borders of the right ventricular myocardium are manually traced at end-diastole on this short-axis cardiac magnetic resonance image. This scan is taken from a patient with idiopathic pulmonary hypertension. Right ventricular dilatation, hypertrophy and increased trabeculation are evident. LV: left ventricle.

(in square centimetres). Integrating the volumetric flow curve over systole gives the stroke volume. This imaging technique has been available for $>20$ yrs [20]. Velocity-encoded imaging has been shown to be a reliable method to measure blood flow in different vessels of the body. Analogous to Doppler echocardiography, this technique allows the calculation of stroke volume, cardiac output, ejection fraction, valvular regurgitant fractions and quantification of cardiac shunts, while mitral and tricuspid transvalvular flow profiles allow the assessment of ventricular diastolic filling patterns (E and A waves). Cardiac output and the pulmonary to systemic flow ratio measured with the use of this technique have been shown to be accurate $[21,22]$. Stroke volume calculated from flow measurements in the pulmonary artery corresponds well with volumetric measurements of the right ventricle in healthy subjects. Phase contrast magnetic resonance flow is less accurate in patients with either cardiac arrhythmia during acquisition or turbulent blood flow; the presence of these is a general limitation of this technique. Of note, even when appropriate methods of acquisition have been used, there can be inaccuracies of flow measurement on some CMR systems caused by background phase errors due to eddy currents or uncorrected concomitant gradients.

\section{Contrast-enhanced CMR imaging}

Gadolinium is a contrast agent utilised in magnetic resonance scanning. It has seven unpaired electrons in its outer shell, and it hastens T1 relaxation, thereby increasing signal in the area of interest. Gadolinium alone is cytotoxic, but not if chelated with diethylenetriamine pentaacetic acid. It has similar pharmacokinetic properties to iodinated X-ray contrast but with minimal nephrotoxicity and anaphylaxis risk. Attention has been drawn, however, to recent reports identifying a possible link 
between exposure to gadolinium-containing agents used in patients with end-stage renal disease and a rare, potentially life-threatening, condition referred to as nephrogenic systemic fibrosis. Regulatory authorities advise caution in the administration of gadolinium-containing agents in renally impaired patients.

In addition to evaluating the first-pass transit of gadolinium contrast, images can be obtained $10-15$ min later, in a pseudoequilibrium phase. Gadolinium is avidly retained in abnormal myocardial regions, resulting in shortened $\mathrm{T} 1$ and increased signal intensity. The bright areas on the resulting images are described as areas of delayed contrast enhancement (DCE). DCE is not biologically specific and has been described in a variety of illnesses. Myocardial infarction, fibrosis and inflammation have all been shown to result in DCE using gadolinium as an i.v. contrast agent [23-27].

\section{Magnetic resonance pulmonary circulation}

Several methods have been proposed for MRI imaging of the pulmonary vasculature, both with and without the use of gadolinium. Three-dimensional gadolinium-enhanced magnetic resonance angiography (MRA) is now the most commonly applied. Contrast-enhanced MRA utilises three-dimensional ultrafast imaging sequences (T1 weighted) after i.v. injection of gadolinium and uses the first pass of this contrast agent [28]. Limitations of MRA include a lower spatial resolution and longer breath-hold when compared with CT.

Preliminary protocols are being developed to image lung perfusion into the diseased lung. These will allow for quantitative analysis of lung perfusion. This technique may allow for perfusion/functional assessment before and after disease targeted therapy.

\section{CMR ASSESSMENT OF PULMONARY HYPERTENSION}

It is becoming increasingly recognised that the right ventricle and the pulmonary vasculature should be approached, both diagnostically and therapeutically, as a unit in patients with pulmonary hypertension.

\section{Cardiac morphology and function}

\section{Ventricular volumes}

Right ventricular end-diastolic and end-systolic volumes are significantly elevated in pulmonary hypertension patients when compared with control subjects [11, 29-31]. These increased volumes represent the dilatation of the right ventricle (fig. 2). Right ventricular ejection fraction is significantly impaired in pulmonary hypertension compared with healthy subjects [29, 30, 32]. Right ventricular stroke volume [29] and right ventricular cardiac output are significantly reduced in patients with pulmonary hypertension compared with healthy control subjects [32]. A recent CMR study of 64 patients with idiopathic pulmonary arterial hypertension confirmed that a large right ventricular volume and a low stroke volume measured at baseline were strong independent predictors of mortality and treatment failure [33].

Using CMR imaging, left ventricular end-diastolic volume, left ventricular stroke volume and left ventricle peak filling rate (expressed as left ventricular end-diastolic volume per second) were significantly smaller in patients with pulmonary hypertension compared with healthy controls [29]. A reduced left ventricular end-diastolic volume at baseline predicts a poor outcome [33]. A CMR study by VONK NOORDEGRAAF et al. [32] compared patients with pulmonary hypertension secondary to emphysema and healthy controls. A significantly reduced left ventricular ejection fraction was demonstrated in the emphysematous patients and, especially, in those without right ventricular hypertrophy. Decreased left ventricular volumes can be explained by the increased PVR, which limits right ventricular stroke volume and, therefore, the volume available for left ventricle filling. Left ventricular septal bowing further reduces the left ventricular volume in early diastole, thus limiting the left ventricle filling process during the most important phase of rapid filling. GAN et al. [34] investigated the contribution of direct right to left ventricular interaction to left ventricle filling and stroke volume in pulmonary arterial hypertension patients and controls using CMR. They confirmed a close relationship between left ventricular enddiastolic volume and stroke volume, and concluded that ventricular interaction mediated by the interventricular septum (IVS) impairs left ventricle filling, contributing to a decreased stroke volume.

\section{Ventricular mass}

CMR has confirmed a significantly higher right ventricle mass in patients with pulmonary hypertension compared with healthy volunteers (fig. 2). Right ventricular hypertrophy is a consequence of the increased pulmonary afterload [13]. The left ventricular mass does not differ significantly from normal values in pulmonary hypertension patients [13]. A study by SABA et al. [35] of 26 patients who underwent CMR and echocardiography examination shortly after RHC showed that a ventricular mass index (VMI) $>0.6$ (obtained by dividing right ventricle mass by left ventricle mass) had a sensitivity of $84 \%$ and specificity of $71 \%$ for detecting pulmonary hypertension of various aetiologies. The VMI was more accurate than echocardiography in diagnosing pulmonary hypertension and demonstrated excellent correlation $(r=0.81)$ with mean $P$ pa determined during right heart catheterisation [35]. This correlation was superior to that obtained from right ventricle mass alone. A recent, larger study by RoELEVELD et al. [36], however, showed a much weaker correlation between the VMI and mean $P$ pa $(\mathrm{r}=0.56)$, although the VMI was found to be the best among five different CMR-based methods for the estimation of mean $P$ pa.

\section{Interventricular septal configuration}

Cine CMR images show characteristic right ventricular wall motion changes in pulmonary hypertension patients. Distortion of the normal shape of the IVS has been reported in situations of right ventricular pressure and/or volume overload [37]. In the presence of increased systolic pressure in the right ventricle, the IVS flattens and may bow toward the left ventricle (fig. 2). Severe left ventricular septal bowing is often considered to be associated with an unfavourable prognosis in pulmonary hypertension [38]. Flattening and bowing of the septum can be expressed quantitatively as curvature, where the curvature is defined as the reciprocal of the radius. In 2005, ROELEVELD et al. [39] investigated whether a relationship existed between septum shape and systolic $P$ pa in patients with pulmonary hypertension. It was concluded that 
systolic Ppa was proportional to septal curvature $(\mathrm{r}=0.77$, $\mathrm{p}<0.001$ ). Maximal distortion of the normal septal shape was found during the right ventricular relaxation phase. The cause of the leftward septum displacement appeared to be a pressure excess in the right ventricle relative to the left ventricle. Data obtained from 39 subjects showed a systolic $P$ pa $>67 \mathrm{mmHg}$ might be expected if left ventricular septal bowing is seen.

\section{Right ventricular diastolic function}

Diastolic function has been shown to be abnormal in diseases affecting the left ventricle. Often, diastolic dysfunction is an early sign of ventricular dysfunction and is currently being targeted therapeutically. GAN et al. [40] have shown that right ventricular diastolic dysfunction is present in pulmonary hypertension patients and can be relatively easily measured by CMR. Isovolumetric relaxation time (IVRT) may be a marker of right ventricular diastolic dysfunction and might predict burden of disease and clinical outcomes. IVRT correlates positively with both right ventricle mass and PVR, variables that are known to be of critical importance in the evaluation and prognosis of pulmonary hypertension [41]. Perhaps, more importantly, IVRT improves in response to standard therapies known to decrease right ventricular afterload, e.g. oral sildenafil [42]. These data suggest that MRImeasured right ventricular diastolic dysfunction and IVRT

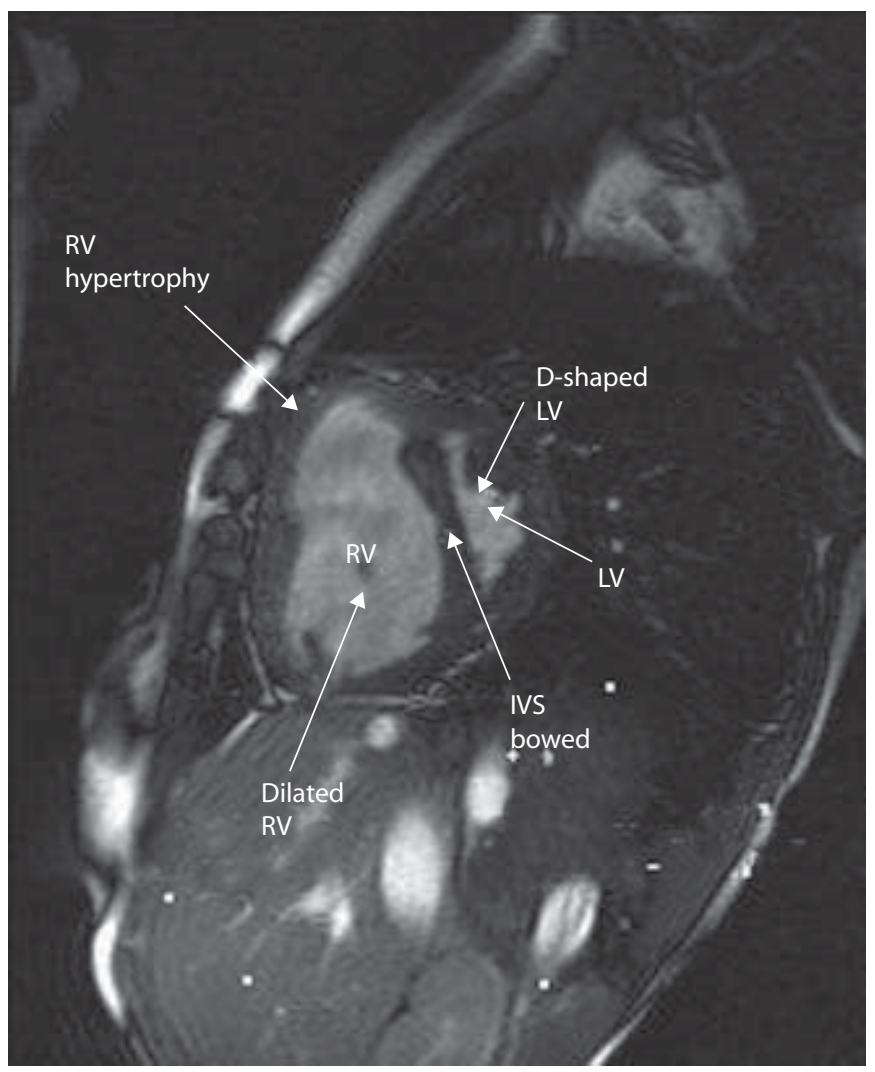

FIGURE 2. Cardiac magnetic resonance (CMR) short-axis image from a patient with pulmonary hypertension. A short-axis cine image at mid-ventricular level in early diastole. The CMR image was acquired from a patient with severe idiopathic pulmonary arterial hypertension. The right ventricle (RV) is grossly dilated and hypertrophied. The distorted interventricular septum (IVS) is bowed towards the left ventricle (LV; D-shaped) owing to right ventricular pressure overload. might be a good surrogate end-point for clinical trials on pulmonary hypertension. This comes at a very good time, where hard end-points, directly relevant to the right ventricle, need to be identified and used in pulmonary hypertension clinical trials; the validity of currently used primary endpoints, such as the 6-min walk test (6MWT), are being challenged [43].

\section{Right ventricular contractility}

Recent advances in magnetic resonance scanner hardware and software have enabled CMR guidance of endovascular catheters under real-time imaging (magnetic resonance fluoroscopy). This CMR approach is a promising tool for assessing right ventricular contractility in the clinical setting [44]. KUEHNE et al. [44] have demonstrated it is possible to combine CMR-guided invasive right ventricular pressure measurements with right ventricular volume values derived from cine CMR and to obtain right ventricular pressure-volume loops. This first study of six patients with early-stage idiopathic pulmonary arterial hypertension (mean \pm SD Ppa $57 \pm 21 \mathrm{mmHg}$ ) and six controls, demonstrated that the right ventricular and left ventricular stroke volumes and cardiac indices were significantly lower, despite higher right ventricular ejection fractions and right ventricular contractility in these patients. CMR-guided RHC was successfully used by the same group to assess the changes in PVR after nitric oxide inhalation in patients with idiopathic pulmonary arterial hypertension [45]. Reduction or elimination of $\mathrm{X}$-ray radiation, added anatomic and functional information available with magnetic resonance, and the relative ease and accuracy of phase contrast magnetic resonance flow quantification may make this technique the method of choice for invasive measurement of PVR. This is a single-centre experience, and major limitations are cost and availability of magnetic resonance-compatible equipment. This procedure is not suitable for serial follow-up owing to its invasive nature.

\section{Contrast-enhanced perfusion CMR}

An interesting pattern of hyperenhancement within the right ventricle is described with delayed-contrast CMR in patients with pulmonary hypertension (fig. 3). This delayed-contrast enhancement pattern has a mid-wall distribution involving the right ventricle septal insertion points and the IVS [46]. A higher degree of enhancement was correlated with poorer right ventricular function and haemodynamics. When contrast enhancement was present in the IVS, it was associated with septal bowing on cine CMR. These data was confirmed by MCCANN et al. [27].

\section{Stress CMR}

Stress testing, by exercise or drug infusion, can be used to determine cardiac reserve. Physical exercise within the confines of the magnet is technically difficult and leads to image degradation. Holverda et al. [47], however, demonstrated that idiopathic pulmonary arterial hypertension patients were unable to significantly increase stroke volume from rest to exercise, using an magnetic resonance-compatible ergometer. Pharmacological CMR stress can be used in patients with congenital heart disease to detect early right ventricular dysfunction. The physiological effects of exercise are imitated by a continuous infusion of a short-acting agent such as dobutamine (a relatively selective $\beta_{1}$-adrenoceptor 


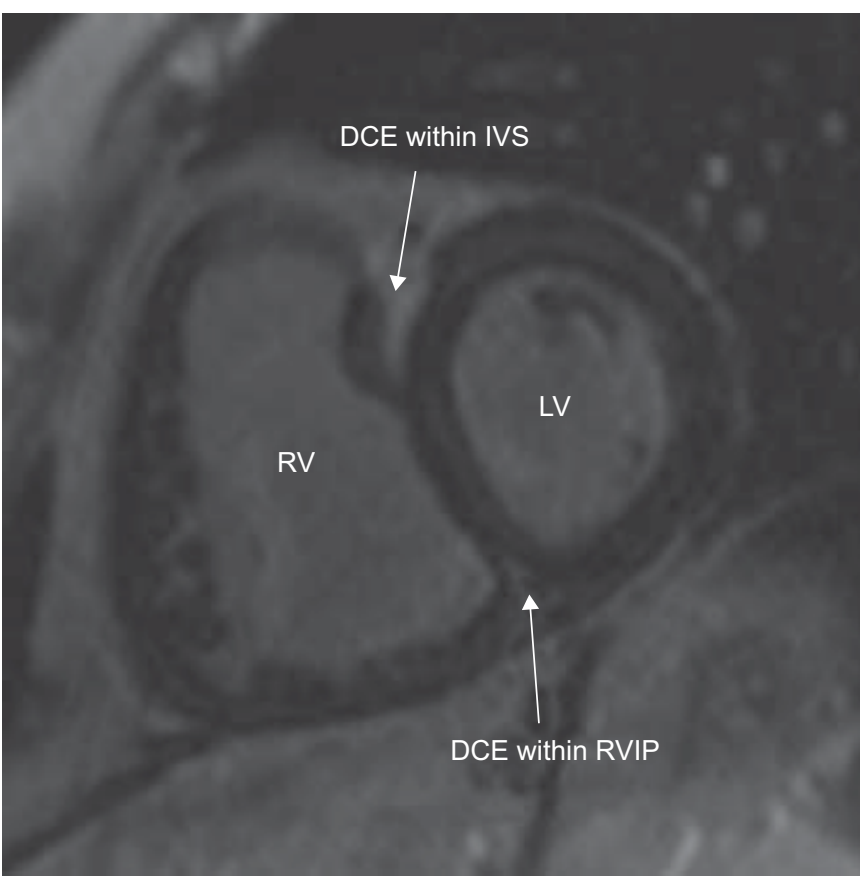

FIGURE 3. Delayed contrast-enhanced cardiac magnetic resonance (CMR) images of a patient with pulmonary hypertension. A contrast-enhanced short-axis CMR cine image was acquired at a basal ventricular level. The delayed-contrast enhancement (DCE) pattern has a mid-wall distribution involving the right ventricular insertion point (RVIP) and the interventricular septum (IVS). Trabeculations and papillary muscles are deliberately included in the analysis, as this has been shown to be a more accurate, although time-consuming, method. RV: right ventricle; LV: left ventricle.

agonist) [48]. Dobutamine has a positive inotropic effect on right ventricular contractility, which can be determined using MRI. To the best of our knowledge, there is no literature regarding pharmacological stress in pulmonary hypertension patients published at present.

\section{Pulmonary circulation in pulmonary hypertension}

CMR flow measurements

Velocity-encoded imaging is another CMR approach for the assessment of pulmonary hypertension. The analysis of these images enables the description of changes or irregularities of pulmonary blood flow in pulmonary hypertension. Previous studies using this technique have found highly inhomogeneous velocity profiles, a large volume of retrograde flow and decreased distensibility of the main pulmonary artery in patients with pulmonary hypertension [49, 50]. From the quantitative analysis of the pulmonary flow profile, noninvasive indices (e.g. acceleration time, defined as time from onset of flow to the peak velocity, and acceleration volume) have been derived for the assessment of PVR [51]. Peak blood flow velocity in the main pulmonary artery is lower in patients with pulmonary hypertension and shows inverse correlation with mean Ppa and PVR. When examined in patients with CTEPH, values after pulmonary endarterectomy were significantly higher than before surgical intervention, but did not reach normal range [52]. A significant reduction of peak velocity in both right and left pulmonary arteries was observed in patients with pulmonary hypertension secondary to cystic fibrosis [53].
As the study revealed no change in the flow of the main pulmonary artery, it was concluded that early and subtle changes of pulmonary haemodynamics are first noticeable in the periphery of the pulmonary arterial system. CMR could, therefore, be the method of choice for detection of early haemodynamic change before right ventricle function is altered.

In the setting of PHT, the most important applications of flow analysis include measurement of cardiac output and pulmonary to systemic flow measurements in the estimation of right-to-left and left-to-right shunts. Results from MRI flow measurements correspond well with thermodilution data obtained but cardiac output determination using velocity-encoded MRI offers advantages over the thermodilution method. It is noninvasive and the measurements depend less on changes in stroke volume from one cardiac cycle to another, because it averages over many cardiac cycles. In addition, the values are not influenced by tricuspid regurgitation to such a great extent.

Right ventricular stroke volume can be calculated as the difference between end-diastolic and end-systolic right ventricular volumes, or by the measurement of volumetric flow in the main pulmonary artery employing phase contrast velocity mapping. Stroke volume, calculated from flow measurements in the pulmonary artery and from volumetric measurements of the right ventricle, corresponds well in healthy controls, and show little divergence in patients with mild tricuspid regurgitation. However, with considerable tricuspid regurgitation (e.g. pulmonary hypertension patients), the volumetric stroke volume overestimates the actual stroke volume [29], because it is impossible to differentiate between the volume that moves back through the tricuspid valve and forward though the pulmonary valve. Flow measurement values are considered more reliable.

\section{Distensibility of pulmonary artery}

Pulmonary artery distensibility measured by CMR (expressed as per cent variation and calculated according to the following equation) was found to be significantly lower in pulmonary hypertension patients than it was in normal subjects $(8 \%$ versus $23 \%$ ) [49]

Pulmonary artery distensibility $=$

$(($ maximal area - minimal area $) /$ minimal area $) \times 100$

A recent pilot, prospective study performed by JARDIM et al. [54] indicated that the noninvasive assessment of pulmonary artery distensibility by magnetic resonance reflected the acute response pattern in idiopathic pulmonary hypertension patients. Pulmonary artery distensibility was significantly higher in responders to an acute vasodilator test during invasive haemodynamic evaluation. GAN et al. [55] have recently demonstrated that proximal pulmonary artery stiffness (in terms of area distensibility and noninvasively assessed relative area change by $\mathrm{CMR}$ ) predicted mortality in patients with pulmonary hypertension.

\section{CMR pulmonary angiography}

The typical findings of CTEPH (intraluminal webs and bands, vessel cut-offs, and organised thrombus) are well demonstrated by pulmonary MRA and can be seen in vessels to segmental level. Beyond the segmental level, the higher spatial resolution of conventional angiography makes it superior. 
Surgical intervention is largely limited to proximal and segmental vessels, and in a study by KREITNER et al. [52], contrast-enhanced MRA correctly predicted surgical success in 33 out of 34 patients. The study demonstrated that threedimensional contrast-enhanced MRA performed equally as well as X-ray pulmonary angiography for the visualisation of segmental pulmonary vessels (533 out of 533 segments), was slightly worse for subsegmental vessels (681 versus 733 segments), but was superior for the depiction of the central origin of thromboembolic material. Pulmonary MRA may be combined in the same examination with a variety of cine techniques to gauge cardiac function and flow. Contrastenhanced MRA should identify patients with CTEPH that delineate typical findings and are potential candidates for surgery.

\section{CMR pulmonary perfusion imaging}

OHNO et al. [56] have demonstrated that three-dimensional dynamic contrast-enhanced MRI has the potential for assessment of disease severity in pulmonary hypertension patients. This technique showed significant differences in pulmonary blood flow and mean transit time between healthy and pulmonary hypertension subjects.

\section{PPA ESTIMATION BY CMR}

Repeated measurements of $P$ pa are sometimes used to assess disease progression in pulmonary hypertension. Echocardiography is safe and widely available but has limitations, as previously discussed [57]. MRI has been proposed to be an accurate alternative for echocardiography in estimating $P$ pa. Investigators have attempted to use CMR as a noninvasive means of estimating mean $P$ pa but none have reported any advantages over echocardiography. Several estimators based on different MRI techniques have been described in recent years, including acceleration time (the time of onset of forward flow to the moment of maximum flow velocity in the main pulmonary artery), acceleration time/ejection time ratio, pulse wave velocity, cross-sectional area of the main pulmonary artery and ventricular mass index. Right ventricular end-diastolic wall thickness has been shown to correlate well with mean $P$ pa in idiopathic pulmonary arterial hypertension and some cases of secondary pulmonary hypertension [58, 59]. A linear relationship between right ventricle mass and mean $P$ pa has been described for idiopathic pulmonary arterial hypertension [13]. The ratio of the main pulmonary artery diameter over descending aortic diameter has also been shown to correlate with mean $P$ pa in pulmonary hypertension. The VMI was found to be the best among five different CMR-based methods for the estimation of $P$ pa and similar to echocardiography ( $r=0.55$ using the modified Bernoulli equation and peak tricuspid regurgitation velocity), but not accurate enough to replace RHC in clinical practice [36]. A computed method for the noninvasive magnetic resonance assessment of pulmonary hypertension has been elaborated, in which a combination of physical variables, including main pulmonary artery blood flow velocity at peak systole, maximal systolic main pulmonary artery cross sectional area and biophysical parameters including patient height, weight and heart rate were used to estimate $P$ pa $[60,61]$.

\section{FOLLOW-UP OF PATIENTS WITH PULMONARY HYPERTENSION}

CMR is increasingly used in patients with pulmonary hypertension for the evaluation of pathological and functional changes in the heart and pulmonary circulation. CMR provides a direct evaluation of right ventricular size, mass, morphology and function [62]. Normal ranges have been established [13, 50]. CMR findings in right ventricular failure include right ventricular dilatation, tricuspid regurgitation, right ventricular hypertrophy, interventricular septal flattening or paradoxical motion, and change in chamber morphology from a normal crescent shape to a more concentric form. Noninvasive assessments of blood flow (including stroke volume and cardiac output) and distensibility in the pulmonary arteries can be made [63-65]. There is good correlation between RHC and magnetic resonance, suggesting that magnetic resonance data could be used as a surrogate of right heart haemodynamics [64].

Pulmonary hypertension experts gathered in 2007 at the End Points Meeting held in Turnberry, UK. Physicians currently rely on the World Health Organization (WHO) functional class, 6MWT, biological markers (brain natriuretic peptide (BNP) levels), echocardiography and RHC to follow up patients with pulmonary hypertension. These investigations have acknowledged limitations. The question of which endpoints are most relevant in the assessment of pulmonary hypertension has been the topic of intense discussion. The WHO functional class has been an important end-point in clinical trials of pulmonary hypertension, although the assignment of patients to categories is subject to the bias of investigators, which limits its usefulness as an end-point. The $6 \mathrm{MWT}$ is a submaximal exercise test which can be performed by patients who are incapable of tolerating maximal exercise testing [66]. The 6MWT has been used widely as a primary end-point in clinical trials, but flaws have been highlighted in its performance. The $6 \mathrm{MWT}$ must be performed correctly using the appropriate guidelines [66]. There are concerns that the 6min walk distance (6MWD) is affected by a number of factors other than pulmonary hypertension including age, sex, height, weight and musculoskeletal conditioning [67]. Furthermore, it has been shown that the 6MWD can improve considerably with rehabilitation measures alone [43]. Echocardiography is the most well established and accessible imaging modality for follow-up of patients with pulmonary hypertension. Doppler echocardiography is suitable for serial assessments, although it has some limitations, as discussed previously in the present article. Serial measurement of plasma NT-proBNP (Nterminal-pro-BNP) has great attractions as an end-point. Its presence in the blood is related to right ventricular dysfunction, it is simple to measure and relatively inexpensive. Some remarkable relationships between plasma BNP/NT-proBNP and various elements of right ventricular dysfunction have been shown $[46,68,69]$. It would appear that BNP/NTproBNP measurement is a dynamic measurement reflecting the current state of the right ventricle. An increase in NTproBNP over time reflects right ventricular dilatation concomitant to hypertrophy and deterioration of systolic function [69]. We await the results of large-scale studies to determine the role of BNP in the assessment and management of patients with pulmonary hypertension. The normalisation of measures 

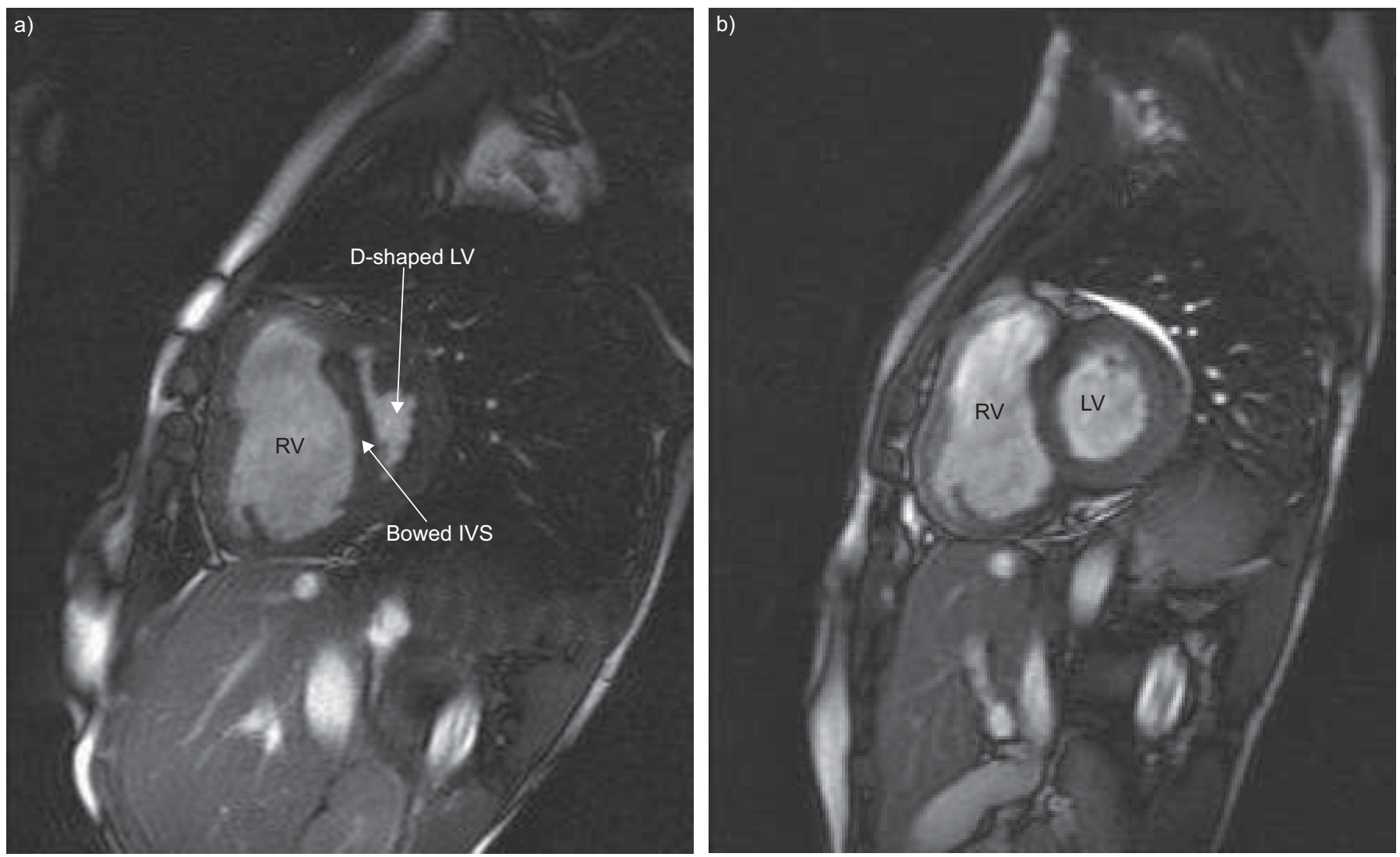

FIGURE 4. Pre- and post-treatment cardiac magnetic resonance (CMR) imaging. A 14-yr-old male presented with dyspnoea and exertional syncope. Idiopathic pulmonary arterial hypertension was diagnosed. a) Baseline short-axis mid-ventricular CMR image demonstrating a grossly dilated right ventricle (RV) with pronounced bowing of the interventricular septum (IVS) compromising the left ventricle (LV). Oral bosentan and anticoagulation therapy were commenced. There was considerable functional improvement. b) A CMR scan repeated at 6 months demonstrated a dramatic improvement in cardiac morphology.

of cardiovascular haemodynamics would be an ideal endpoint. However, resting haemodynamics improve only marginally in most patients, even when their clinical response appears to be excellent [70], and do not reflect changes that may occur with exercise. Clinical improvement, therefore, is only partly related to a modification of resting haemodynamics in most patients. Furthermore, RHC is an invasive procedure that is not ideal for serial evaluation.

It has been suggested that characteristics for an ideal marker in pulmonary hypertension might include [71]: 1) it should be heart or lung specific; 2) it should be abnormal in pulmonary hypertension; 3) sample collection should be simple; 4) the marker should be easy to measure; 5) values should be reproducible; 6) values should follow the course of the disease (i.e. increasing if patients deteriorate and falling if patients improve); and 7) abnormal values should be indicative of a poor survival.

CMR imaging fulfils these stated characteristics. As discussed, modern CMR protocols provide us with abundant information regarding the ventricular myocardium and pulmonary vasculature. Right ventricular volumes, muscle mass and functional parameters, including stroke volume, ejection fraction and cardiac output, differ significantly in pulmonary hypertension compared to healthy subjects. CMR imaging is easily performed by trained MRI technicians/physicians and the majority of patients tolerate this noninvasive investigation well. Manual planimetry of the myocardium and flow analysis is simple to perform and reproducible although time-consuming at present. Sequential MRI is the optimal tool to monitor therapeutic effects on vascular remodelling and right heart performance. CMR-derived right ventricle functional parameters correlate well with established haemodynamic parameters of prognostic significance. Although RHC remains the definitive assessment of pulmonary hypertension at present, the noninvasive evaluation of cardiac morphology and function and of the pulmonary circulation is a new and promising application for CMR imaging.

\section{CMR AS AN END-POINT}

MRI is gaining a dominant role as the reference method for clinical trials assessing longitudinal changes in left ventricular function after therapeutic interventions [72-74]. The accuracy and reproducibility of CMR in assessing cardiac morphological and functional variables leads to low interstudy variability, which translates into a significant reduction in sample sizes required to test the efficacy of therapeutic interventions. It is expected that the number of clinical trials using CMR parameters as study end-points will increase considerably in the future. Ultimately, however, patient outcome is the relevant clinical issue. Future effort should be directed toward testing whether changes in cardiac variables as measured by magnetic 
resonance indeed translate into differences in patient outcome. An example of CMR-measured improvement is seen in figure 4 .

Deterioration of right ventricular function at follow-up examinations indicates an unfavourable prognosis because functional impairment of the right ventricle is the major factor in disease progression and decline in life expectancy [75]. VAN WOLFEREN et al. [33] performed a longitudinal CMR study which confirmed right ventricular dilatation and a decrease in stroke volume and left ventricular diastolic volume are strong predictors of treatment failure and death at follow-up. Medical therapies or surgical interventions may stop, or even reverse, this process, and the improvement of right ventricular function could be detected by MRI.

At present, there are few studies in pulmonary hypertension which have utilised CMR as an end-point so far. Changes in right ventricle mass, function and pulmonary artery blood flow have been demonstrated by CMR following lung transplantation in several studies [74, 76, 77]. In 2003, MiCHELAKIS et al. [78] performed a small nonrandomised pilot study of five patients with pulmonary hypertension to investigate the effect of sildenafil $50 \mathrm{mg}$ t.i.d. Right ventricle mass was utilised as an end-point. Sildenafil significantly reduced right ventricle mass and increased the right ventricular stroke volume as measured by CMR. The pathological septal shift towards the left ventricle was reversed by longterm sildenafil therapy. In a prospective study of pulmonary hypertension patients with prostacyclin therapy, the significant increase in right ventricular stroke volume (pulmonary arterial flow analysis) corresponded well with functional improvement (WHO functional class and 6MWT) [30]. The functional and morphological effects of pulmonary endarterectomy in patients with CTEPH was assessed by KREITNER et al. [52] using a combination of three-dimensional gadolinium contrastenhanced MRA, cine CMR and velocity-encoded CMR. CMR demonstrated surgical success in 33 out of the 34 patients, with improvement of the initially depressed right ventricular ejection fraction, which correlated with the decrease in mean $P$ pa and disappearance of septal bowing in $68 \%$ of patients after surgery. In a comparison study between sildenafil and bosentan, right ventricle mass (measured by CMR) did not change after 3 months of bosentan treatment, whereas sildenafil reduced right ventricle mass [79]. It was demonstrated by CMR that the addition of sildenafil reversed right ventricular dilatation and hypertrophy in patients receiving treatment [80]. More recently, 16 patients with pulmonary arterial hypertension were assessed by $\mathrm{CMR}$ at baseline and after 12 months treatment with bosentan [81]. After treatment, cardiac index, PVR and 6MWT distance increased. There was a trend towards improvement in right ventricular stroke volume $(p=0.08)$, although there was no change in right ventricular ejection fraction or right ventricular end-diastolic volume, as determined by CMR.

\section{CONCLUSIONS}

CMR imaging enables a unique combination of morphological and functional assessment of the right ventricle and pulmonary circulation. CMR has emerged over recent years as the gold standard for detailed study of the right ventricle and has become an established modality for the physiological assessment of pulmonary hypertension patients in cross-sectional studies, longitudinal follow-up studies and clinical trials of therapy. We anticipate that MRI will increasingly be utilised as the primary modality for combined anatomic and functional assessments that enable more complete and efficient evaluation of patients with pulmonary hypertension. CMR is currently being used as an end-point in the multinational European Union-funded Framework 6 EURO-MR project for pulmonary hypertension. We wait to see whether the promise of CMR as a successful end-point is fulfilled.

\section{REFERENCES}

1 Rubin LJ. Primary pulmonary hypertension. N Engl J Med 1997; 336: 111-117.

2 Runo JR, Loyd JE. Primary pulmonary hypertension. Lancet 2003; 361: 1533-1544.

3 Simonneau G, Galie N, Rubin LJ, et al. Clinical classification of pulmonary hypertension. J Am Coll Cardiol 2004; 43, 12: Suppl. S, 5S-12S

4 Kukulski T, Voigt JU, Wilkenshoff UM, et al. A comparison of regional myocardial velocity information derived by pulsed and color Doppler techniques: an in vitro and in vivo study. Echocardiography 2000; 17: 639-651.

5 Currie PJ, Seward JB, Chan KL, et al. Continuous wave Doppler determination of right ventricular pressure: a simultaneous Doppler-catheterization study in 127 patients. J Am Coll Cardiol 1985; 6: 750-756.

6 Denton CP, Cailes JB, Phillips GD, et al. Comparison of Doppler echocardiography and right heart catheterization to assess pulmonary hypertension in systemic sclerosis. $\mathrm{Br}$ J Rheumatol 1997; 36: 239-243.

7 Arcasoy SM, Christie JD, Ferrari VA, et al. Echocardiographic assessment of pulmonary hypertension in patients with advanced lung disease. Am J Respir Crit Care Med 2003; 167: 735-740.

8 Fedullo PF, Auger WR, Kerr KM, et al. Chronic thromboembolic pulmonary hypertension. N Engl J Med 2001; 345: 1465-1472.

9 Hoeper MM, Lee SH, Voswinckel R, et al. Complications of right heart catheterization procedures in patients with pulmonary hypertension in experienced centers. J Am Coll Cardiol 2006; 48: 2546-2552.

10 Ley S, Kreitner KF, Fink C, et al. Assessment of pulmonary hypertension by CT and MR imaging. Eur Radiol 2004; 14: 359-368.

11 Boxt LM, Katz J, Kolb T, et al. Direct quantitation of right and left ventricular volumes with nuclear magnetic resonance imaging in patients with primary pulmonary hypertension. J Am Coll Cardiol 1992; 19: 1508-1515.

12 Doherty NE 3rd, Fujita N, Caputo GR, et al, Measurement of right ventricular mass in normal and dilated cardiomyopathic ventricles using cine magnetic resonance imaging. Am J Cardiol 1992; 69: 1223-1228.

13 Katz J, Whang J, Boxt LM, et al. Estimation of right ventricular mass in normal subjects and in patients with primary pulmonary hypertension by nuclear magnetic resonance imaging. J Am Coll Cardiol 1993; 21: 1475-1481.

14 Pattynama PM, Willems LN, Smit AH, et al. Early diagnosis of cor pulmonale with MR imaging of the right ventricle. Radiology 1992; 182: 375-379.

15 Suzuki J, Caputo GR, Masui T, et al. Assessment of right ventricular diastolic and systolic function in patients with 
dilated cardiomyopathy using cine magnetic resonance imaging. Am Heart J 1991; 122: 1035-1040.

16 Bottini PB, Carr AA, Prisant LM, et al. Magnetic resonance imaging compared to echocardiography to assess left ventricular mass in the hypertensive patient. Am J Hypertens 1995; 8: 221-228.

17 Grothues F, Smith GC, Moon JC, et al. Comparison of interstudy reproducibility of cardiovascular magnetic resonance with two-dimensional echocardiography in normal subjects and in patients with heart failure or left ventricular hypertrophy. Am J Cardiol 2002; 90: 29-34.

18 Semelka RC, Tomei E, Wagner S, et al. Normal left ventricular dimensions and function: interstudy reproducibility of measurements with cine MR imaging. Radiology 1990; 174: 763-768.

19 Grothues F, Moon JC, Bellenger NG, et al. Interstudy reproducibility of right ventricular volumes, function, and mass with cardiovascular magnetic resonance. Am Heart J 2004; 147: 218-223.

20 Nayler GL, Firmin DN, Longmore DB. Blood flow imaging by cine magnetic resonance. J Comput Assist Tomogr 1986; 10: 715-722.

21 Beerbaum $\mathrm{P}$, Korperich $\mathrm{H}$, Barth $\mathrm{P}$, et al. Noninvasive quantification of left-to-right shunt in pediatric patients: phase-contrast cine magnetic resonance imaging compared with invasive oximetry. Circulation 2001; 103: 2476-2482.

22 Robertson MB, Kohler U, Hoskins PR, et al. Quantitative analysis of PC MRI velocity maps: pulsatile flow in cylindrical vessels. Magn Reson Imaging 2001; 19: 685-695.

23 Holman ER, van Jonbergen HP, van Dijkman PR, et al. Comparison of magnetic resonance imaging studies with enzymatic indexes of myocardial necrosis for quantification of myocardial infarct size. Am J Cardiol 1993; 71: 1036-1040.

24 Laissy JP, Messin B, Varenne O, et al. MRI of acute myocarditis: a comprehensive approach based on various imaging sequences. Chest 2002; 122: 1638-1648.

25 Lima JA, Judd RM, Bazille A, et al. Regional heterogeneity of human myocardial infarcts demonstrated by contrastenhanced MRI. Potential mechanisms. Circulation 1995; 92: 1117-1125.

26 Teraoka K, Hirano M, Ookubo H, et al. Delayed contrast enhancement of MRI in hypertrophic cardiomyopathy. Magn Reson Imaging 2004; 22: 155-161.

27 McCann GP, Gan CT, Beek AM, et al. Extent of MRI delayed enhancement of myocardial mass is related to right ventricular dysfunction in pulmonary artery hypertension. AJR Am J Roentgenol 2007; 188: 349-355.

28 Uematsu H, Ohno Y, Hatabu H. Recent advances in magnetic resonance perfusion imaging of the lung. Top Magn Reson Imaging 2003; 14: 245-251.

29 Hoeper MM, Tongers J, Leppert A, et al. Evaluation of right ventricular performance with a right ventricular ejection fraction thermodilution catheter and MRI in patients with pulmonary hypertension. Chest 2001; 120: 502-507.

30 Roeleveld RJ, Vonk-Noordegraaf A, Marcus JT, et al. Effects of epoprostenol on right ventricular hypertrophy and dilatation in pulmonary hypertension. Chest 2004; 125 : 572-579.

31 Vonk-Noordegraaf A, Marcus JT, Holverda S, et al. Early changes of cardiac structure and function in COPD patients with mild hypoxemia. Chest 2005; 127: 1898-1903.
32 Vonk Noordegraaf A, Marcus JT, Roseboom B, et al. The effect of right ventricular hypertrophy on left ventricular ejection fraction in pulmonary emphysema. Chest 1997; 112: 640-645.

33 van Wolferen SA, Marcus JT, Boonstra A, et al. Prognostic value of right ventricular mass, volume, and function in idiopathic pulmonary arterial hypertension. Eur Heart $J$ 2007; 28: 1250-1257.

34 Gan CT, Lankhaar JW, Marcus JT, et al. Impaired left ventricular filling due to right-to-left ventricular interaction in patients with pulmonary arterial hypertension. Am J Physiol Heart Circ Physiol 2006; 290: H1528-H1533.

35 Saba TS, Foster J, Cockburn M, et al. Ventricular mass index using magnetic resonance imaging accurately estimates pulmonary artery pressure. Eur Respir J 2002; 20: 1519-1524.

36 Roeleveld RJ, Marcus JT, Boonstra A, et al. A comparison of noninvasive MRI-based methods of estimating pulmonary artery pressure in pulmonary hypertension. J Magn Reson Imaging 2005; 22: 67-72.

37 King ME, Braun H, Goldblatt A, et al. Interventricular septal configuration as a predictor of right ventricular systolic hypertension in children: a cross-sectional echocardiographic study. Circulation 1983; 68: 68-75.

38 D'Alonzo GE, Barst RJ, Ayres SM, et al. Survival in patients with primary pulmonary hypertension. Results from a national prospective registry. Ann Intern Med 1991; 115: 343-349.

39 Roeleveld RJ, Marcus JT, Faes TJ, et al. Interventricular septal configuration at MR imaging and pulmonary arterial pressure in pulmonary hypertension. Radiology 2005; 234: 710-717.

40 Gan CT, Holverda S, Marcus JT, et al. Right ventricular diastolic dysfunction and the acute effects of sildenafil in pulmonary hypertension patients. Chest 2007; 132: 11-17.

41 McLaughlin VV, Presberg KW, Doyle RL, et al. Prognosis of pulmonary arterial hypertension: ACCP evidence-based clinical practice guidelines. Chest 2004; 126: 78S-92S.

42 Michelakis E, Tymchak W, Lien D, et al. Oral sildenafil is an effective and specific pulmonary vasodilator in patients with pulmonary arterial hypertension: comparison with inhaled nitric oxide. Circulation 2002; 105: 2398-2403.

43 Mereles D, Ehlken N, Kreuscher S, et al. Exercise and respiratory training improve exercise capacity and quality of life in patients with severe chronic pulmonary hypertension. Circulation 2006; 114: 1482-1489.

44 Kuehne T, Yilmaz S, Steendijk P, et al. Magnetic resonance imaging analysis of right ventricular pressure-volume loops: in vivo validation and clinical application in patients with pulmonary hypertension. Circulation 2004; 110: 2010-2016.

45 Kuehne T, Yilmaz S, Schulze-Neick I, et al. Magnetic resonance imaging guided catheterisation for assessment of pulmonary vascular resistance: in vivo validation and clinical application in patients with pulmonary hypertension. Heart 2005; 91: 1064-1069.

46 Blyth KG, Groenning BA, Martin TN, et al. Contrast enhanced-cardiovascular magnetic resonance imaging in patients with pulmonary hypertension. Eur Heart J 2005; 26: 1993-1999. 
47 Holverda S, Gan CT, Marcus JT, et al. Impaired stroke volume response to exercise in pulmonary arterial hypertension. J Am Coll Cardiol 2006; 47: 1732-1733.

48 Tulevski, II, Lee PL, Groenink M, et al, Dobutamine-induced increase of right ventricular contractility without increased stroke volume in adolescent patients with transposition of the great arteries: evaluation with magnetic resonance imaging. Int J Card Imaging 2000; 16: 471-478.

49 Bogren HG, Klipstein RH, Mohiaddin RH, et al. Pulmonary artery distensibility and blood flow patterns: a magnetic resonance study of normal subjects and of patients with pulmonary arterial hypertension. Am Heart J 1989; 118: 990-999.

50 Kondo C, Caputo GR, Masui T, et al. Pulmonary hypertension: pulmonary flow quantification and flow profile analysis with velocity-encoded cine MR imaging. Radiology 1992; 183: 751-758.

51 Mousseaux E, Tasu JP, Jolivet O, et al. Pulmonary arterial resistance: noninvasive measurement with indexes of pulmonary flow estimated at velocity-encoded MR imaging - preliminary experience. Radiology 1999; 212: 896-902.

52 Kreitner KF, Ley S, Kauczor HU, et al. Chronic thromboembolic pulmonary hypertension: pre- and postoperative assessment with breath-hold MR imaging techniques. Radiology 2004; 232: 535-543.

53 Ley S, Puderbach M, Fink C, et al. Assessment of hemodynamic changes in the systemic and pulmonary arterial circulation in patients with cystic fibrosis using phase-contrast MRI. Eur Radiol 2005; 15: 1575-1580.

54 Jardim C, Rochitte CE, Humbert M, et al. Pulmonary artery distensibility in pulmonary arterial hypertension: a MRI pilot study. Eur Respir J 2007; 29: 476-481.

55 Gan CT, Lankhaar JW, Westerhof N, et al. Noninvasively assessed pulmonary artery stiffness predicts mortality in pulmonary arterial hypertension. Chest 2007; 132: 1906-1912.

56 Ohno Y, Hatabu H, Murase K, et al. Primary pulmonary hypertension: 3D dynamic perfusion MRI for quantitative analysis of regional pulmonary perfusion. AJR Am J Roentgenol 2007; 188: 48-56.

57 Yock PG, Popp RL. Noninvasive estimation of right ventricular systolic pressure by Doppler ultrasound in patients with tricuspid regurgitation. Circulation 1984; 70: 657-662.

58 Bouchard A, Higgins CB, Byrd BF 3rd, et al. Magnetic resonance imaging in pulmonary arterial hypertension. Am J Cardiol 1985; 56: 938-942.

59 Saito H, Dambara T, Aiba M, et al. Evaluation of cor pulmonale on a modified short-axis section of the heart by magnetic resonance imaging. Am Rev Respir Dis 1992; 146: 1576-1581.

60 Laffon E, Vallet C, Bernard V, et al. A computed method for noninvasive MRI assessment of pulmonary arterial hypertension. J Appl Physiol 2004; 96: 463-468.

61 Lankhaar JW, Vonk Noordegraaf A, Marcus JT. A computed method for noninvasive MRI assessment of pulmonary arterial hypertension. J Appl Physiol 2004; 97: 794.

62 Lorenz CH, Walker ES, Morgan VL, et al. Normal human right and left ventricular mass, systolic function, and gender differences by cine magnetic resonance imaging. $J$ Cardiovasc Magn Reson 1999; 1: 7-21.
63 Marcus JT, Vonk Noordegraaf A, Roeleveld RJ, et al. Impaired left ventricular filling due to right ventricular pressure overload in primary pulmonary hypertension: noninvasive monitoring using MRI. Chest 2001; 119: 1761-1765.

64 Paz R, Mohiaddin RH, Longmore DB. Magnetic resonance assessment of the pulmonary arterial trunk anatomy, flow, pulsatility and distensibility. Eur Heart J 1993; 14: 1524-1530.

65 Tardivon AA, Mousseaux E, Brenot F, et al. Quantification of hemodynamics in primary pulmonary hypertension with magnetic resonance imaging. Am J Respir Crit Care Med 1994; 150: 1075-1080.

66 ATS statement: guidelines for the six-minute walk test. Am J Respir Crit Care Med 2002; 166: 111-117.

67 Rich S. The current treatment of pulmonary arterial hypertension: time to redefine success. Chest 2006; 130: 1198-1202.

68 Nagaya N, Nishikimi T, Okano Y, et al. Plasma brain natriuretic peptide levels increase in proportion to the extent of right ventricular dysfunction in pulmonary hypertension. J Am Coll Cardiol 1998; 31: 202-208.

69 Gan CT, McCann GP, Marcus JT, et al. NT-proBNP reflects right ventricular structure and function in pulmonary hypertension. Eur Respir J 2006; 28: 1190-1194.

70 Castelain V, Chemla D, Humbert M, et al. Pulmonary artery pressure-flow relations after prostacyclin in primary pulmonary hypertension. Am J Respir Crit Care Med 2002; 165: 338-340.

71 Peacock A, Naeije R, Galie N, et al. End points in pulmonary arterial hypertension: the way forward. Eur Respir J 2004; 23: 947-953.

72 Longmore DB, Klipstein RH, Underwood SR, et al. Dimensional accuracy of magnetic resonance in studies of the heart. Lancet 1985; 1: 1360-1362.

73 Moon JC, Lorenz CH, Francis JM, et al. Breath-hold FLASH and FISP cardiovascular MR imaging: left ventricular volume differences and reproducibility. Radiology 2002; 223: 789-797.

74 Moulton MJ, Creswell LL, Ungacta FF, et al. Magnetic resonance imaging provides evidence for remodeling of the right ventricle after single-lung transplantation for pulmonary hypertension. Circulation 1996; 94: II312-II319.

75 Sitbon $\mathrm{O}$, Humbert $\mathrm{M}$, Nunes $\mathrm{H}$, et al. Long-term intravenous epoprostenol infusion in primary pulmonary hypertension: prognostic factors and survival. J Am Coll Cardiol 2002; 40: 780-788.

76 Frist WH, Lorenz CH, Walker ES, et al. MRI complements standard assessment of right ventricular function after lung transplantation. Ann Thorac Surg 1995; 60: 268-271.

77 Mohiaddin RH, Paz R, Theodoropoulos S, et al. Magnetic resonance characterization of pulmonary arterial blood flow after single lung transplantation. J Thorac Cardiovasc Surg 1991; 101: 1016-1023.

78 Michelakis ED, Tymchak W, Noga M, et al. Long-term treatment with oral sildenafil is safe and improves functional capacity and hemodynamics in patients with pulmonary arterial hypertension. Circulation 2003; 108: 2066-2069.

79 Wilkins MR, Paul GA, Strange JW, et al. Sildenafil versus Endothelin Receptor Antagonist for Pulmonary 
Hypertension (SERAPH) study. Am J Respir Crit Care Med 2005; 171: 1292-1297.

80 van Wolferen SA, Boonstra A, Marcus JT, et al. Right ventricular reverse remodelling after sildenafil in pulmonary arterial hypertension. Heart 2006; 92: 1860-1861.
81 Chin KM, Kingman M, de Lemos JA, et al. Changes in right ventricular structure and function assessed using cardiac magnetic resonance imaging in bosentan-treated patients with pulmonary arterial hypertension. Am J Cardiol 2008; 101: 1669-1672. 